\title{
Distribución geográfica, historia natural y conservación del hurón menor Galictis cuja (Carnivora: Mustelidae) en la Patagonia central, Argentina
}

\section{Geographic distribution, natural history and conservation of the lesser grison Galictis cuja (Carnivora: Mustelidae) from Central Patagonia, Argentina}

\author{
Marcelo Carrera ${ }^{1}$, Marcela Janina Nabte ${ }^{2}$ y Daniel Edgardo Udrizar-Sauthier ${ }^{3 凶}$ \\ 'Facultad de Ciencias Naturales, sede Puerto Madryn, Universidad Nacional de la Patagonia “San Juan Bosco”, Boulevard Brown 3051, (U9120ACF) \\ Puerto Madryn, Chubut, Argentina. \\ ${ }^{2}$ Unidad de Investigación Ecología Terrestre, Centro Nacional Patagónico-CONICET. Casilla de Correo 128, Puerto Madryn 9120 Chubut, Argentina. \\ ${ }^{3}$ Unidad de Investigación Diversidad, Sistemática y Evolución, Centro Nacional Patagónico-CONICET, Casilla de Correo 128, Puerto Madryn 9120 \\ Chubut, Argentina. \\ \dsauthier@cenpat.edu.ar
}

\begin{abstract}
Resumen. El hurón menor, Galictis cuja, tiene una amplia distribución en el territorio patagónico extra-andino, aunque sus registros puntuales son escasos. Este trabajo se desarrolló en la provincia del Chubut, Patagonia Central, Argentina. Aquí se aportan nuevas localidades de registro de G. cuja para esta región; se discuten aspectos de su distribución geográfica y conservación en el Área Natural Protegida Península Valdés (ANP-PV; Patrimonio Natural de la Humanidad) y brevemente se explora la representación de G. cuja en los ensambles de carnívoros del ANP-PV desde el Holoceno tardío hasta la actualidad. Se adicionaron 18 nuevos registros de G. cuja en Patagonia central. Se detectó un conflicto entre los pobladores y hurones, que motiva la caza de estos últimos. Se verificó un aparente incremento de abundancia de G. cuja en los últimos miles de años, concomitante con la extinción regional o dramática disminución de Lyncodon patagonicus (Carnivora, Mustelidae).
\end{abstract}

Palabras clave: carnívoros, conservación, distribución geográfica, Holoceno tardío, Patagonia.

\begin{abstract}
The Lesser Grison, Galictis cuja, is a species widely distributed in extra-Andean Patagonia, although its records are scarce. This work was carried out in Chubut province, Central Patagonia, Argentina. Here we report new occurrence localities of G. cuja for this region; we discuss aspects of their geographical distribution and conservation in the Área Natural Protegida Península Valdés (ANP-PV; World Heritage Site) and briefly explores the representation of G. cuja in carnivore assemblages of ANP-PV, since the late Holocene to the present. We added 18 new records of $G$. cuja in Central Patagonia. We detected a conflict between the rural residents and the Lesser Grison, which motivates the hunting of the latter. There was an apparent increase in abundance of G. cuja in the last thousands of years, concomitant with regional extinction or dramatic reduction of Lyncodon patagonicus (Carnivora, Mustelidae).
\end{abstract}

Key words: carnivores, conservation, geographic distribution, Late Holocene, Patagonia.

En la Patagonia continental argentina habitan 3 especies de mustélidos autóctonos, Lontra provocax, Lyncodon patagonicus y Galictis cuja; a las que se adiciona una especie exótica introducida, Mustela vison (Monjeau et al., 1994). La distribución geográfica de $L$. provocax está restringida a sólo 3 áreas aisladas de la Región AndinoPatagónica, siendo la más importante el Parque Nacional Nahuel Huapi (Aued et al., 2003). Mientras que L. patagonicus y G. cuja tienen una amplia distribución en el territorio patagónico extrandino, aunque sus registros puntuales son escasos (cf. Redford y Eisenberg, 1992; Yensen

Recibido: 29 agosto 2011; aceptado: 20 abril 2012 y Tarifa, 2003; Prevosti y Travaini, 2005; Prevosti et al., 2009). Mustela vison fue introducido en criaderos del oeste de Patagonia a mediados del siglo XX con fines peleteros (Pagnoni et al., 1986; Godoy, 1963). Algunos ejemplares fueron liberados y otros escaparon, estableciéndose poblaciones silvestres que colonizaron las márgenes de ambientes fluviales andinos y extrandinos (Previtalli et al., 1998). Los cursos de agua fueron utilizados como corredores, favoreciendo la dispersión de la especie (Pagnoni et al., 1986).

Este trabajo se desarrolló en la provincia del Chubut, Patagonia Central (entre los $42^{\circ}$ y $46^{\circ} \mathrm{S}$; Fig. 1), poniéndose énfasis en el Área Natural Protegida Península Valdés 
(ANP-PV), declarada Patrimonio Natural de la Humanidad por UNESCO.

El objetivo de esta nota es aportar nuevas localidades de registro de Galictis cuja para el área protegida y regiones aledañas; se discuten aspectos de su distribución geográfica y conservación en el ANP-PV y brevemente se explora la representación de G. cuja en relación con $L$. patagonicus en los ensambles de carnívoros del ANP-PV desde el Holoceno tardío (últimos 3000 años) hasta la actualidad.

El ANP-PV se emplaza en el noreste de la provincia del Chubut entre los $42^{\circ} 00^{\prime}-42^{\circ} 45^{\prime}$ S, 63 $65^{\circ}-65^{\circ} 17^{\prime} \mathrm{O}$; abarca una superficie de 400000 ha. Las características climáticas generales del ANP-PV son las dominantes en la región noreste de la Patagonia, modificadas localmente por las interacciones entre la atmósfera y los cuerpos de agua adyacentes (Labraga, 1994). Las precipitaciones se concentran durante la estación fría, desde mayo a septiembre (Paruelo et al., 1998) y son muy escasas, fluctuando entre 200 y $225 \mathrm{~mm}$ de media anual (Barros y Rivero, 1981). La temperatura media anual es de $12.6^{\circ} \mathrm{C}$ (Barros, 1983). Fitogeográficamente están representadas 2 unidades mayores: la Provincia del Monte y el Ecotono Monte-Patagonia (León et al., 1998). El principal uso de la tierra en esta región es la ganadería extensiva ovina (Beeskow et al., 1987).
Este trabajo es el producto de 10 años de estudio y recolecciones que los autores han venido realizando en Patagonia central. Los ejemplares consignados fueron obtenidos en viajes de campo que se llevaron a cabo con el fin de estudiar aspectos taxonómicos y de conservación de la mastofauna patagónica y que son parte medular de las tesis de doctorado de Udrizar-Sauthier (2009) y Nabte (2010). Sólo se brindan localidades de registro de $G$. cuja en las cuales se obtuvieron materiales de referencia o en las que los autores personalmente avistaron a los animales. Los materiales se encuentran depositados en la Colección de Mamíferos del Centro Nacional Patagónico (CNP) y en la Colección Anatómica Marcelo Carrera (MC), ambas colecciones se encuentran en Puerto Madryn, Chubut, Argentina. Otros materiales pertenecen al catálogo de campo de los autores (MJN y DEUS; véase Cuadro 1). Adicionalmente a estos registros, se realizaron encuestas escritas mediante entrevistas personalizadas a pobladores rurales, a razón de un encuestado por establecimiento ganadero $(\mathrm{N}=82)$. Es importante destacar que la totalidad de las entrevistas fueron realizadas por la misma persona asegurándose de unificar criterios, tanto de preguntas como respuestas. Inicialmente se interrogó si la especie se encontraba presente en el establecimiento; cuando la respuesta fue afirmativa, se consultó qué abundancia le asignaba el entrevistado (poco abundante,

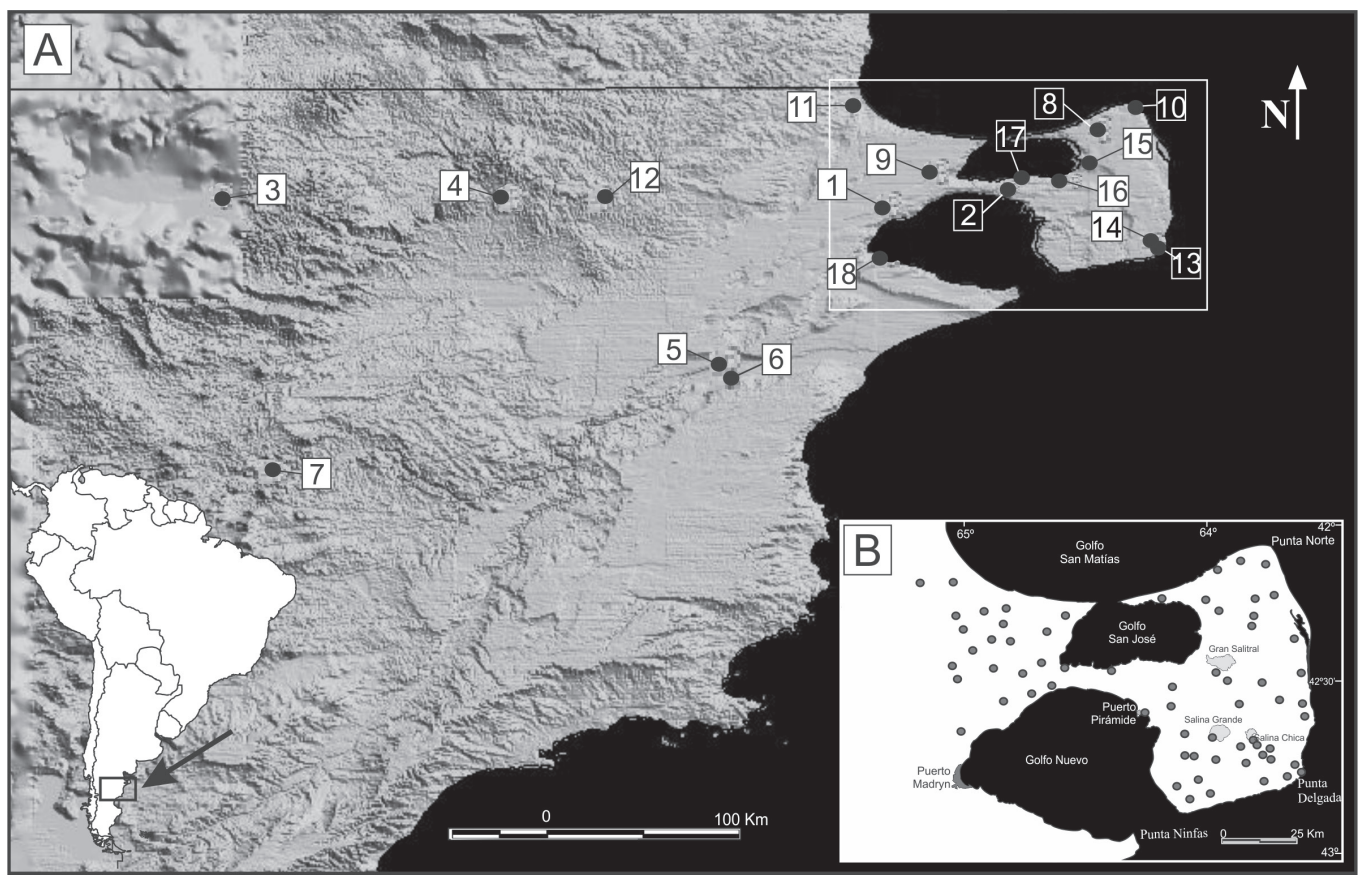

Figura 1. Ubicación geográfica del área de estudio. A, nuevos registros de Galictis cuja en Patagonia central. Los números de las localidades corresponden con los del Cuadro 1; B, Área Natural Protegida Península Valdés, Chubut, Argentina. Ubicación de los establecimientos ganaderos en los cuales se constató la presencia de G. cuja a través de encuestas. 


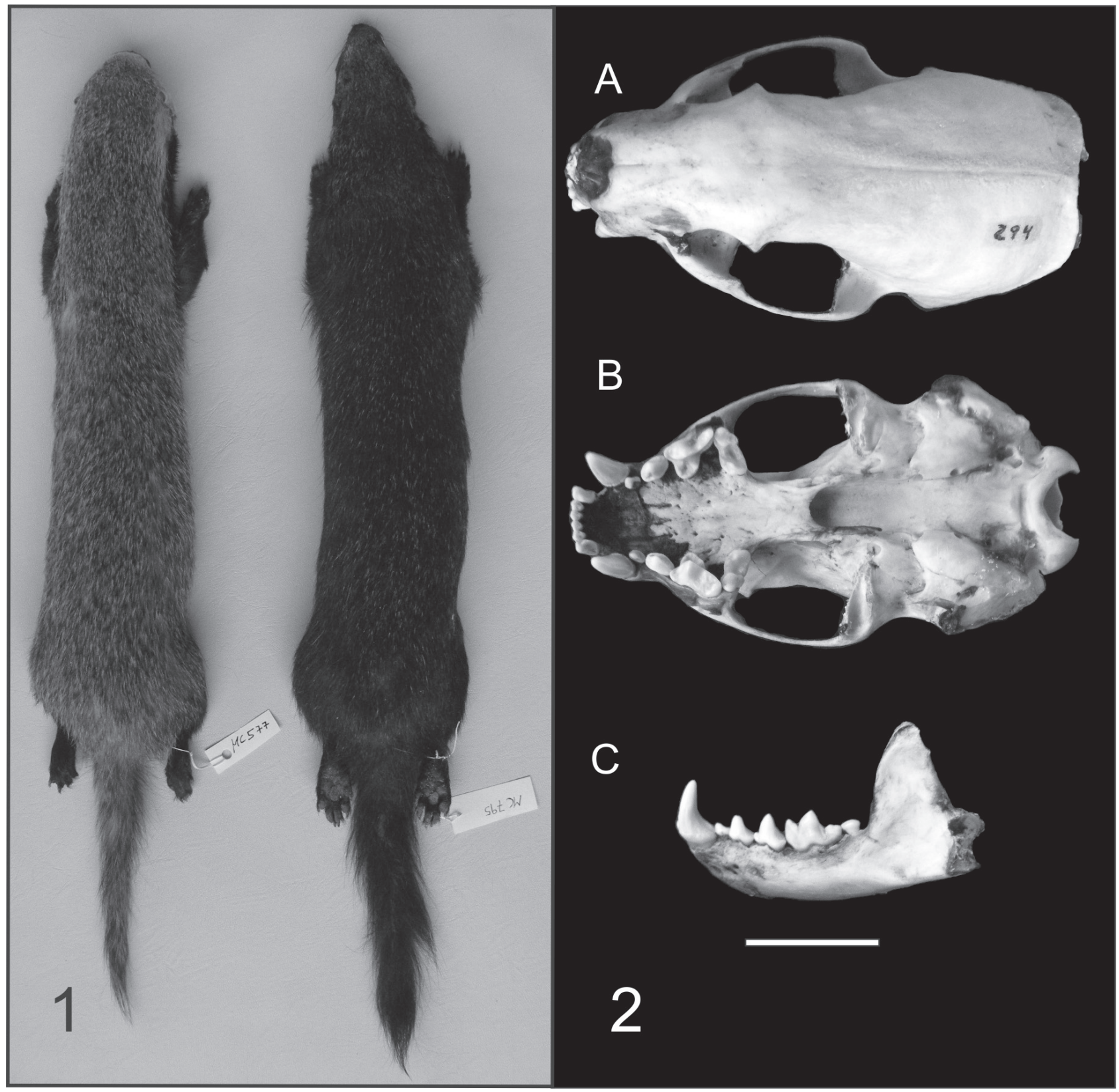

Figura 2. Galictis cuja. Ejemplar mostrando la típica coloración de pelaje (izquierda); ejemplar melánico del Área Natural Protegida Península Valdés, Chubut, Argentina (derecha); A, vista dorsal del cráneo; B, vista ventral del cráneo; C, vista labial de mandíbula izquierda. Escala $20 \mathrm{~mm}$.

abundante o muy abundante). Por último, dependiendo de la antigüedad del encuestado en el establecimiento, se preguntó respecto a la tendencia poblacional en los últimos años, teniendo las siguientes opciones: aumentó mucho, aumentó, se mantuvo igual, disminuyó o disminuyó mucho. Otros datos que se indagaron fueron si la especie se consideraba perjudicial, neutra o beneficiosa para la producción o el bienestar humano, y según la respuesta del entrevistado, se le preguntó cuáles eran los motivos para asignar esa categoría. Por último, se estudió la abundancia de G. cuja en los ensambles de carnívoros del Holoceno tardío (últimos 3000 años), para lo cual se recurrió a la Colección Anatómica Marcelo Carrera en la que se encuentran depositados numerosos ejemplares de carnívoros colectados en yacimientos paleontológicos del ANP-PV. 
Cuadro 1. Localidades de registro de Galictis cuja en el Área Natural Protegida Península Valdés y regiones cercanas de Patagonia central, Argentina.

\begin{tabular}{clccc}
\hline Núm. & Localidad & Latitud $S$ & Longitud O & Referencia \\
\hline 1 & 10.5 km NO conj. RPN ${ }^{\circ}$ y RPN 2 & -42.578000 & -64.928958 & avistaje \\
2 & 11 km E Istmo C. Ameghino & -42.469333 & -64.368417 & MC 795; 795-1 \\
3 & 14 km E de Gan Gan & -42.526222 & -68.114417 & MC 3051 \\
4 & 19 km E Telsen & -42.523528 & -66.742611 & DUS 827 \\
5 & 2.5 km SO Dolavon & -43.323239 & -65.733875 & avistaje \\
6 & 5.5 km S Dolavon & -43.359389 & -65.723444 & CNP 576 \\
7 & Cañadón Carbón & -43.825239 & -67.868133 & avistaje \\
8 & Ea. El Centro & -42.205233 & -63.941869 & MN 408 \\
9 & Ea. La Entrada & -42.415917 & -64.710181 & MN 338 \\
10 & Ea. La Ernestina & -42.080192 & -63.759086 & MN 342 \\
11 & Ea. La Esperanza & -42.109414 & -64.816475 & avistaje \\
12 & Est. María de Las Nieves & -42.524256 & -66.317250 & MC 12 \\
13 & Est. San Félix 1 & -42.743964 & -63.675589 & MC 2204 \\
14 & Est. San Félix 2 & -42.743128 & -63.679303 & MC 1830 \\
15 & Est. San Francisco & -42.340611 & -64.908214 & MC 502 \\
16 & Playa Fracasso & -42.430853 & -64.126533 & MC 294 \\
17 & Playa Larralde & -42.412817 & -64.313800 & MC 246 \\
18 & Playa Paraná & -42.803681 & -64.940794 & avistaje \\
\hline
\end{tabular}

Ea., Estancia; Est., Establecimiento

Los caracteres externos utilizados para discriminar a Galictis cuja de las otras 2 especies de mustélidos presentes en el área de estudio (L. patagonicus y M. vison) fueron: el pelaje dorsal blanco en 2 bandas que no cubre la nuca (Fig. 2); los pelos dorsales, laterales y de la cola de color negro con puntas castaño claro, y la región ventral del cuerpo, miembros, cuello y cabeza, también negros (Parodi, 1937; Cabrera y Yepes, 1960). Desde la región frontal parte una banda de pelos color castaño claro que diverge hacia los laterales del cuello y abarca las orejas. De los ejemplares estudiados, provenientes del ANP-PV, 2 varían en la coloración, son totalmente melánicos (Fig. 2); ambos machos, y pertenecen al mismo grupo familiar. Este fenómeno es común en otros carnívoros (e.g., Leopardus geoffroyi, Conepatus chinga) pero poco documentado en G. cuja (Canevari y Vaccaro, 2007). A partir de caracteres craneanos, G. cuja se diferencia por que su cráneo es de mayor tamaño que el de L. patagonicus y de M. vison, con la superficie fronto-nasal fuertemente convexa, tiene las apófisis supraorbitarias dirigidas en sentido ventroposterior; la constricción post-orbitaria es ancha (media de $19.13 \mathrm{~mm} ; \mathrm{N}=6$ ); el foramen infraorbitario es elíptico, comprimido dorso-ventralmente; las coanas son anchas y las bullas timpánicas presentan menor desarrollo que en las otras 2 especies de mustélidos. La fórmula dentaria es I 3/3, C 1/1, PM 3/3, M 1/2= 34 dientes (Fig. 2).

Se registraron 18 nuevas localidades en Patagonia central, donde se presenta G. cuja; se listan en el Cuadro 1 y su ubicación geográfica se muestra en la Figura 1. Se logró encuestar a los responsables de $82(91 \%)$ de los 90 establecimientos ganaderos presentes en el ANP-PV; no fue posible encuestar a 2 que se localizan en el límite oeste del ANP-PV; algunos se encontraron abandonados, mientras que a otros no fue permitido el acceso. En el 80.5\% $(\mathrm{N}=66)$ de los establecimientos encuestados se constató la presencia de G. cuja (véase Fig. 1B); aunque los pobladores mencionaron que sus registros son poco abundantes. El 37.9\% $(\mathrm{N}=25)$ de los encuestados calificó a G. cuja como una especie perjudicial; el $16.7 \%(\mathrm{~N}=11)$ la consideró una especie que no causa problemas, mientras que el $45.5 \%(\mathrm{~N}=30)$ restante no respondió. Galictis cuja es frecuentemente cazado por los pobladores rurales, quienes aducen varios motivos por los que consideran perjudicial a la especie: 1) "Ataca al ganado ovino, comiéndole la piel y musculatura de la mejilla y extremo anterior del rostro, lo que, si bien no produce la muerte inmediata, el animal no puede alimentarse ni beber agua eficientemente, lo que ocasiona la muerte por inanición" Así lo manifestaron algunos pobladores encuestados $(\mathrm{N}=11)$; aunque en la mayoría de los casos fue un comentario transmitido oralmente y no un hecho directamente observado por ellos. Por este motivo, no se puede confirmar que G. cuja ocasione tal tipo de daño al ganado ovino. Delibes et al. (2003) registran un muy bajo porcentaje de ovinos como parte de la dieta del hurón menor, lo cual podría relacionarse con hábitos carroñeros. Si G. cuja atacara realmente al ganado ovino, las poblaciones de hurones deberían ser más abundantes, teniendo en cuenta que en el ANP-PV hay más de 
200000 cabezas de ganado; 2) "Se acerca a los alojamientos de humanos en busca de los animales de granja para alimentarse (e.g., aves de corral y sus huevos)".

En cuanto a la tendencia poblacional de la especie, el $37.80 \%(\mathrm{~N}=31)$ de los encuestados declararon que se mantiene igual, es decir que no han percibido cambios en el tiempo. Península Valdés es un Área Natural Protegida, por lo tanto está terminantemente prohibida la cacería de fauna silvestre; no obstante, G. cuja es objeto de caza por parte de los pobladores rurales. No existen hasta el momento estudios poblacionales para saber si la extracción de individuos que se hace de esta especie es sustentable en el tiempo. Por otro lado, no se cuenta con un sistema de control eficiente por parte de los guardafaunas del ANP-PV, ya que el personal se encuentra asignado principalmente a tareas de control de las áreas costeras donde se emplazan los distintos puntos turísticos (colonias de aves y mamíferos marinos), dejando relegado gran parte del ambiente terrestre del ANP-PV. Asimismo, se ha constatado la presencia de razas de perros muy agresivos para la fauna silvestre (e.g., galgo, bull terrier). Estas razas no están necesariamente destinadas al trabajo de campo y sólo cumplen con su papel de mascotas, por lo que se podría prescindir de las mismas en el ANP-PV. Por lo tanto, es de suma importancia que se desarrollen pautas de manejo acordes con la problemática que enfrenta $G$. cuja, minimizando el conflicto hurón-humano y maximizando las estrategias de conservación de la especie (Nabte, 2010).

En cuanto a la distribución geográfica de G. cuja en Patagonia central (provincia del Chubut), hasta el momento sólo se conocían 3 registros de esta especie (Redford y Eisenberg, 1992; Yensen y Tarifa, 2003; Wilson y Mittermeier, 2009); 2 se dieron a conocer para el oeste del Chubut y pertenecen a la subespecie G. c. cuja; el tercero se encuentra en el ANP-PV (Estancia La Corona; Daciuk, 1974) y corresponde a la subespecie G. c. huronax (Yensen y Tarifa, 2003). El registro de Estancia La Corona era el único que se conocía para la subespecie en Patagonia. Todos los registros nuevos documentados en este trabajo pertenecen al área de distribución de G. c. huronax, por lo que se incrementa considerablemente el número de localidades en Patagonia donde está presente esta subespecie. Prevosti y Travaini (2005) reseñaron la distribución austral de G. cuja; estos autores aportaron nuevas localidades y discutieron los registros previos en el extremo sur de Sudamérica. A pesar de la nueva información suministrada por Prevosti y Travaini (2005) persistía un extenso vacío en la distribución de esta especie entre el registro de Daciuk (1974) en el ANP-PV y Caleta Olivia, en el norte de la provincia de Santa Cruz. Esta falta de información es parcialmente subsanada por el presente trabajo a partir del registro de G. cuja en 3 localidades intermedias (véase Fig. 1; locs. 5, 6, 7).
En las últimas décadas se ha expandido notablemente la distribución geográfica de Mustela vison, especialmente sobre la cuenca del río Chubut (ca. $1300 \mathrm{~km}$ desde sus nacientes al este de Los Andes hasta su desembocadura en el océano Atlántico). La distribución geográfica de este mustélido introducido es simpátrica con la de G. cuja en extensas áreas de este río; esto plantea un interrogante en cuanto a la interacción de las 2 especies de mustélidos y sobre todo a la existencia de competencia que pudiera afectar la especie autóctona (Delibes et al., 2003).

El estudio de los ensambles de carnívoros fósiles del ANP-PV asignados al Holoceno tardío muestra que $G$. cuja era una especie poco abundante y que representaba el $2.06 \%$ del total del ensamble registrado (número mínimo de individuos $=242$ ). Por otro lado, si consideramos la otra especie de mustélido presente en los ensambles fósiles, Lyncodon patagonicus, se observa que representa el $17.35 \%$ del ensamble, siendo proporcionalmente más abundante. La relación entre ambas especies en el ensamble de carnívoros del Holoceno tardío es de 1:9. Este no es un dato menor, dado que actualmente las evidencias disponibles indican que L. patagonicus habría desaparecido del ANP-PV (Nabte, 2010; Carrera et al. datos no publicados); lo cual estaría reflejando un aparente recambio entre ambas especies de mustélidos en los últimos miles a cientos de años. Las causales de este fenómeno están siendo exploradas y podrían tener su correlato con pulsos climáticos, como la Pequeña Edad del Hielo (Villalba, 1990), que se han traducido en Patagonia como cambios ambientales. Recientemente, en Patagonia central se han registrado extinciones regionales y disminución en la abundancia de especies de pequeños mamíferos vinculados a las estepas patagónicas (Udrizar-Sauthier, 2009), las cuales son reemplazadas en abundancia por otras especies de las estepas arbustivas más áridas de la Provincia Fitogeográfica del Monte (León et al., 1998). Para el caso de las especies de mustélidos, podría estar ocurriendo algo similar: $L$. patagonicus, vinculada a las estepas herbáceas más frías, se retrae a localidades occidentales y G. cuja aumenta su frecuencia en las estepas arbustivas del ANP-PV.

Los autores desean expresar su gratitud a Walter Udrizar-Sauthier por su asistencia durante las tareas de campo. A Silvina Rodríguez, Roberto Williams, Víctor Huenteslá, Luis Coliner y a Gabriela (guardafauna del ANP-PV), por su ayuda en la colecta y procesamiento de los ejemplares estudiados. A Florencia del Castillo-Bernal, Analía Andrade, Germán Cheli, Cecilia Reeves y Sergio Saba, por la ayuda desinteresada en la realización de las encuestas en el campo. A los pobladores rurales por recibirnos en sus hogares y permitirnos hacerles las entrevistas. A Tana por su compañia y amistad. Este trabajo está parcialmente financiado por la Administración del ANP-PV y CONICET. 


\section{Literatura citada}

Aued, M. B., C. Chehebar, G. Porro, D. W. Macdonald y M. H. Cassini. 2003. Environmental correlates of the distribution of southern river otters Lontra provocax at different ecological scales. Oryx 37:413-421.

Barros, V. 1983. Atlas del potencial eólico de la Patagonia. Contribución 69. Centro Nacional Patagónico, Puerto Madryn, Chubut. 80 p.

Barros, V. R. y M. M. Rivero. 1981. Mapas de probabilidad de precipitación de la provincia de Chubut. Subsecretaría de estado de Ciencia y Tecnología, Consejo Nacional de Investigaciones Científicas y Técnicas, Centro Nacional Patagónico, Puerto Madryn, Chubut.

Beeskow, A. M., H. F. Del Valle y C. M. Rostagno. 1987. Los sistemas fisiográficos de la región árida y semiárida de la provincia de Chubut. Secretaría de Ciencia y Técnica, Delegación Regional Patagonia, San Carlos de Bariloche, Río Negro. 168 p.

Cabrera, A. y J. Yepes. 1960. Mamíferos sudamericanos, segunda edición. EDIAR, Buenos Aires. 187 p.

Canevari, M. y O. Vaccaro. 2007. Guía de mamíferos del sur de América del Sur, Literature of Latin America, Buenos Aires. $413 \mathrm{p}$.

Daciuk, J. 1974. Notas faunísticas y bioecológicas de Península Valdés y Patagonia. XII. Mamíferos colectados y observados en la Península Valdés y zona litoral de los golfos San José y Nuevo (Provincia de Chubut, República Argentina). Physis 33:23-39.

Delibes, M., A. Travaini, S. C. Zapata y F. Palomares. 2003. Alien mammals and the trophic position of the lesser grison (Galictis cuja) in Argentinean Patagonia. Canadian Journal of Zoology 81:157-162.

Godoy, J. C. 1963. Fauna silvestre. Evaluación de los recursos naturales de la Argentina, tomo VIII. Consejo Federal de Inversiones. Buenos Aires. 527 p.

Labraga, J. C. 1994. On extreme winds in Pampa del Castillo Plateau, Patagonia Argentina, with reference to wind farms settlement. Journal of Applied Meteorology 33:85-95.

León, R. J. C., D. Bran, M. Collantes, J. M. Paruelo y A. Soriano. 1998. Grandes unidades de vegetación de la Patagonia extra andina. In Ecosistemas patagónicos, M. Oesterheld, M. R. Aguiar y J. M. Paruelo (eds.). Ecología Austral 8:125-144.
Monjeau, J. A., N. Bonino y S. L. Saba. 1994. Annotated checklist of the living land mammals in Patagonia, Argentina. Mastozoología Neotropical 1:143-156.

Nabte, M. J. 2010. Desarrollo de criterios ecológicos para la conservación de mamíferos terrestres en Península Valdés. Tesis, Doctorado Facultad de Ciencias Exactas y Naturales. Universidad Nacional de Mar del Plata, Buenos Aires. 233 p.

Pagnoni, G. O., J. Garrido y M. R. Martin. 1986. Impacto económico y ambiental del visón. Informe técnico. Consejo Nacional de Investigaciones Científicas y Técnicas/ Dirección de Fauna Silvestre, Provincia de Chubut [Argentina].

Parodi, R. 1937. Fauna argentina I, Mamíferos. Sud Americana, Buenos Aires. 235 p.

Paruelo, J. M., A. Beltrán, E. Jobbágy, O. E. Sala y R. A. Golluscio. 1998. The climate of Patagonia: general patterns and controls on biotic processes. In Ecosistemas patagónicos, M. Oesterheld, M. R. Aguiar y J. M. Paruelo (eds.). Ecología Austral 8:85-101.

Previtali, A., M. Cassini y D. W. Macdonald. 1998. Habitat use and diet of American mink (Mustela vison) in Argentinian Patagonia. Journal of Zoology 246:482-486.

Prevosti, F. J., P. Teta y U. F. J. Pardiñas. 2009. Distribution, natural history, and conservation of the Patagonian Weasel Lyncodon patagonicus. Small Carnivore Conservation 41:29-34.

Prevosti, F. J. y A. Travaini. 2005. New records of Galictis cuja (Molina, 1782) (Carnivora, Mustelidae) in Southern Patagonia. Mammalian Biology 70:317-320.

Redford, K. H. y J. F. Eisenberg. 1992. Mammals of the Neotropics: the Southern cone: 2. Chile, Argentina, Uruguay, Paraguay. University of Chicago Press, Illinois. 460 p.

Udrizar-Sauthier, D. E. 2009. Los micromamíferos y la evolución ambiental durante el Holoceno en el río Chubut (Chubut, Argentina). Tesis, doctorado Facultad de Ciencias Naturales y Museo, Universidad Nacional de La Plata, Buenos Aires. $329 \mathrm{p}$.

Villalba, R. 1990. Climatic fluctuations in northern Patagonia during the last 1000 years as inferred from tree-ring records. Quaternary Research 34:346-360.

Wilson, D. E. y R. A. Mittermeier. 2009. Handbook of the Mammals of the World. Vol. 1. Carnivores. Lynx, Barcelona. 728 p.

Yensen, E. y T. Tarifa. 2003. Galictis cuja. Mammalian Species 728:1-8. 